\title{
Identificación molecular de la microbiota gastrointestinal del lechón lactante
}

\section{Molecular identification of the gastrointestinal microbiota of the suckling pig}

Fabian-Dominguez, Fredy 1,2,3[0000-0003-3577-5896]; Vásquez-Rojas, Lourdes $^{1,2[0000-0002-0202-9451]}$; Baylon-Cuba, Miluska ${ }^{1,2[0000-0003-1103-345 X] \text {; }}$ López-Flores, Alicia ${ }^{3[0000-0002-4679-6353]}$ y Mialhe, Eric ${ }^{2[0000-0002-5498-4362]}$

${ }^{1}$ Universidad Nacional de Tumbes, Tumbes, Perú

${ }^{2}$ Incabiotec, Tumbes, Perú

${ }^{3}$ Universidad Nacional de San Martín, Tarapoto, Perú

ffabiandunsm.edu.pe

Resumen. La resistencia de microorganismos patógenos a los antibióticos y la posibilidad de residuos de antibióticos en los productos de origen animal provocan una atención creciente, siendo necesario el uso de alternativas potenciales como bacterias benéficas con carácter probiótico para reemplazar los antibióticos en la dieta de los animales. La metodología fue el aislamiento de bacterias ácido lácticas del tracto gastro intestinal de un lechón lactante, seguidamente se realizó la purificación bacteriana en medio de cultivo MRS, extracción de ADN, y en base de las secuencias del $16 \mathrm{~S}$ ADNr fue amplificado por PCR con iniciadores universales. En el análisis bioinformático por el algoritmo de BLAST del National Center for Biotechnology Information se identificaron molecularmente, Lactobacillus farcimenis, Weissella sp, en el estómago; Lactobacillus brevis, Pediococcus pentosaceus, en el intestino delgado y en el intestino grueso, Pediococcus pentosaceus y Lactobacillus plantarum. En conclusión, existe una diversidad de Lactobacillus en el tracto gastrointestal del porcino, siendo un gran potencial como alternativa a los antibióticos en la alimentación y la inmunomodulación del sistema inmune del animal.

Citar como: FabianDominguez, F., VásquezRojas, L., Baylon-Cuba, M., López-Flores, A. \& Mialhe, E. (2021). Identificación molecular del microbiota gastrointestinal del lechón lactante. Revista de Veterinaria y Zootecnia Amazónica, 1(1), 22-30. https://doi.org/10.51252/re vza.v1i1.136

Recibido: 12/04/2021

Aceptado: 15/06/2021

Publicado: 28/07/2021
Palabras clave: farmacología, microorganismos, probióticos, porcinos

Abstract. The resistance of pathogenic microorganisms to antibiotics and the possibility of antibiotic residues in animal products are attracting increasing attention. Being necessary the use of potential alternatives such as beneficial bacteria with a probiotic character to replace antibiotics in the diet of animals. The methodology was the isolation of lactic acid bacteria from the gastrointestinal tract of a suckling pig, followed by bacterial purification in MRS culture medium, DNA extraction, and based on the $16 \mathrm{~S}$ rDNA sequences it was amplified by PCR with universal primers. In the bioinformatic analysis of the National Center for Biotechnology Information, Lactobacillus farcimenis, Weissella sp, in stomach; Lactobacillus brevis, Pediococcus pentosaceus, in the small intestine and in the large intestine, Pediococcus pentosaceus and Lactobacillus plantarum. In conclusion, there is a diversity of Lactobacillus in the gastrointestal tract of the pig, with great potential as an alternative to antibiotics in feeding and immunomodulation of the animal's immune system.

Keywords: microorganisms, pharmacology, probiotics, swine 


\section{$1 \quad$ Introducción}

Los antibióticos son usados como promotores de crecimiento y prevención de enfermedades en producción animal (1), como aditivo en pienso para los animales, contribuyendo a una mejora de la eficiencia económica; sin embargo, el uso excesivo de antibiótico se ha convertido en un problema de salud, tanto para la producción como para el consumidor. En efecto, el uso continuo de antibiótico, es el responsable de los residuos en productos cárnicos y consecuentemente, el riesgo de aumentar la resistencia bacteriana de bacterias patógenas de los animales y de importancia en salud humana (2,3). Como alternativa al uso de antibióticos, los probióticos han mostrado ser una solución con múltiples ventajas tanto para la salud animal como para mejorar la productividad (4).

El microbiota intestinal cumple actividades biológicas que el hospedero carece como, metabolismo de nutrientes, el buen desarrollo de la fisiología del sistema digestivo y la estimulación del sistema inmunitario (5). En animales sanos, la composición del microbiota intestinal permanece en un estado relativamente estable. Los factores de estrés, tanto internos (alergias) o externos (alimentación, condición de cría, mal uso de antibióticos, enfermedades) pueden desencadenar cambios en el microbiota intestinal, conduciendo a un desequilibrio del sistema de defensa y aparición de microorganismos patógenos, causantes de desórdenes digestivos, baja producción y probabilidad de muerte (6).

La función de los probióticos es de mantener la micro población intestinal en un estado estable y prevenir la proliferación de microorganismos patogénicos (7). Además, pueden ser beneficios en términos de mejor absorción de nutrientes, estimulación del crecimiento, aumento de peso y mejora del factor de conversión (8). En diversas investigaciones se han reportado que estas bacterias pueden suprimir la diarrea (9), disminuir la intolerancia a lactosa (10), y complicaciones post operatorias (11), posee actividad antimicrobiana (12), acción anti cancerígena colon rectal $(13,14)$, reduce síntomas irritables del intestino (15), y previene la inflamación y enfermedades del intestino (16).

Varios géneros de bacteria pueden ser usados como probióticos; Lactobacillus, Pediococcus, Bacteroides, Bifidobacterium, Bacillus, Streptococcus y Escherichia coli (17). La naturaleza de las bacterias Gram positivas tiene propiedades competitivas frente a bacterias patógenas en general de tipo Gram negativas (18). Las bacterias probióticas que han sido estudiada en su mayoría Bacillus (Lactobacillus y Bacillus subtilis) muestra gran potencia (19).

Lactobacillus es un componente esencial de la microbiota gastrointestinal de porcinos $(20,21)$, el más representativo es Lactobacillus amylovorus abundante en lechones lactantes $(22,23)$. Muchos microorganismos a menudo están implicados en diarrea post destete como E.coli 
enterotoxigenica (24)(25). En la suplementación alimenticia con Lactobacillus ha demostrado beneficio de la salud intestinal del lechón lactante destetado $(26,27)$.

El éxito de los probióticos se debe a las siguientes funciones (28). Ser un habitante normal de la microflora intestinal. Moderado o fuerte habilidad de adherencia y mejora de la inmunidad de la mucosa intestinal. Resistencia a un número de antibióticos, coccidiostatos, sales biliares y tolerancia para un shock ácido de $\mathrm{pH}$ 2.0-2.5. Un espectro de inhibición o la prevención de agentes patógenos en el tracto gastrointestinal. Las investigaciones han mostrado que Lactobacillus es una especie ideal. Esto puede actuar como un probiótico en mejorar la producción, controlando los microorganismos patogénicos y reduciendo la diarrea en porcinos, especialmente para recién destetado (29).

La búsqueda de una mejor comprensión de cómo operan los probióticos ha catalizado un enorme interés en los procesos de biotecnología molecular que subyacen a las interacciones huésped-microbio. La percepción de los mecanismos de acción de probióticos no sólo puede ayudar a mejorar la credibilidad del concepto de probióticos, sino también para fomentar el desarrollo de nuevas estrategias para la prevención o tratamiento de enfermedades gastrointestinales y autoinmunes (30). El objetivo del estudio fue caracterizar molecularmente el microbiota del tracto gastrointestinal del lechón destetado.

\section{Materiales y Métodos}

\subsection{Material biológico}

Se realizó una selección al azar de un lechón lactante, de una camada de 12 lechones de dos semanas de edad, condición normal de salud de la granja porcina en Tumbes.

\subsection{Aislamiento bacteriano del tracto gastrointestinal}

Las muestras de estómago, intestino delgado e intestino grueso del lechón lactante fueron colectados en medio de cultivo MRS, pH: 5.5 e incubado a $37{ }^{\circ} \mathrm{C}$ por 48 horas, para el aislamiento se realizó la dilución $1 / 40$, posteriormente 100 ul de la dilución fue dispensado dentro del medio cultivo sólido MRS e incubado a $37{ }^{\circ} \mathrm{C}$ por 24 horas. Las colonias bacterianas fueron contadas y subcultivadas. La bacteria purificada, diferenciada por tinción Gram, y almacenada a $-20^{\circ} \mathrm{C}$ con una solución de glicerol al $15 \%$ del volumen final bacteriano.

\subsection{Extracción de ADN genómico bacteriano}

Se procedió a tomar $1.2 \mathrm{ml}$ de medio de cultivo liquido MRS en un tubo de $1.5 \mathrm{ml} \mathrm{y}$ microcentrífugado a $10000 \mathrm{rpm}$ por $2 \mathrm{~min}$. Posteriormente se eliminó el sobrenadante y se resuspende el sedimento en 500 ul de la solución PBS 1X estéril. Se lleva a centrifugar a 10000 rpm por 2 min, se elimina el sobrenadante añadiendo luego 200 ul de la solución TE (Tris 
1M/0.1M EDTA). Luego llevar a ebullición por 10 minutos e inmediatamente colocar sobre hielo por $5 \mathrm{~min}$ centrifugar a $10000 \mathrm{rpm}$ por $1 \mathrm{~min}$. Transferir el sobrenadante a otro microtubo y se le agrega $1 \mathrm{ul}$ de ARNasa y llevar a $65^{\circ} \mathrm{C}$ por 15 minutos y almacenar.

\subsection{PCR amplificación del gen $16 \mathrm{~S} \mathrm{ADNr}$}

La amplificación del gen 16S ADNr fue realizada mediante la técnica de PCR convencional. Para cada reacción se tomó 2.5 ul de buffer 10X, 1 ul de cloruro de magnesio 50 Mm, 0.1 unidad de taq polimerasa (invitrogen), 0.5 ul de Dntps a $10 \mathrm{mM}, 0.6$ ul de cada primer a 15 pmol, el juego de primer 16S ADNr foward F518: (CCAGCAGCCGCGGTAATACG), revers R800: (TACCAGGGTATCTAATCC), 17.6 ul de agua libre de nucleasas y 2 ul de ADN extraído en una reacción final de 25 ul. La programación para el termociclador, temperatura 94 ${ }^{\circ} \mathrm{C}$ por 6 minutos, $94{ }^{\circ} \mathrm{C}$ por 30 segundos, $58{ }^{\circ} \mathrm{C}$ por 45 segundos, $72{ }^{\circ} \mathrm{C}$ por 1 minuto, $72{ }^{\circ} \mathrm{C}$ por 4 minutos y $4{ }^{\circ} \mathrm{C}$ por 10 horas, por 33 ciclos. Los datos de secuencias serán comparados con la secuencia bases de Gen Bank (National Center for Biotechnology Information) usando el algoritmo BLAST.

\section{$3 \quad$ Resultados y discusiones}

\subsection{Bacteria ácido lácticas aisladas en el tracto gastrointestinal del lechón lactante}

Las bacterias aisladas del estómago e intestino delgado crecieron a las 48 horas, intestino grueso a las 24 horas a temperatura de $37^{\circ} \mathrm{C}$ en incubación, se aisló 2 bacterias de estómago, 2 intestino delgado, 2 intestino grueso.

\subsection{Caracterización molecular de bacterias ácido lácticas}

La identidad de todas las cepas bacterianas fue analizado e identificado en la secuencia de $16 \mathrm{~S}$ ADNr fue identificado como, Lactobacillus farcimenis, Weissella sp, aislados del estómago; Lactobacillus brevis, Pediococcus pentosaceus aislado del intestino delgado y Pediococcus pentosaceus, Lactobacillus plantarum, aislado del intestino grueso. La secuencia ADN de identidad 16S ADNr fue 98 a 100\% (ver Tabla 1)

Tabla 1.

Porcentaje de homología de las bacterias identificadas molecularmente

\begin{tabular}{llr}
\hline Tracto gastrointestinal & \multicolumn{1}{c}{ Bacteria } & $\begin{array}{c}\text { Porcentaje de } \\
\text { homología }\end{array}$ \\
\hline Estómago & Lactobacillus farceminis & $98 \%$ \\
& Weissella sp & $99 \%$ \\
Intestino delgado & Pediococcus pentosaceus & $99 \%$ \\
& Lactobacillus brevis & $100 \%$ \\
Intestino grueso & Pediococcus pentosaceus & $99 \%$ \\
& Lactobacillus plantarum & $100 \%$ \\
\hline
\end{tabular}


Existen abundantes microorganismos en el tracto gastrointestinal del porcino. Los Lactobacillus están presentes en el intestino como uno de los microorganismos predominantes (31). Nuestro resultado concuerda con (6), en donde el número de crecimiento de colonias bacterianas sobre agar MRS incremento de posterior a anterior en el intestino. Indicando que el medio ambiente posterior del intestino es mejor para el crecimiento de bacterias tipo Lactobacillus que el medio ambiente anterior, debido al bajo pH en el estómago, sales biliares en el intestino y oxígeno en las heces. Estos resultados concuerdan con (32), pero el número de colonias es variable debido al tipo de crianza de porcinos, dieta y medio ambiente.

Los Lactobacillus en el tracto gastrointestinal del porcino cumplen un rol importante en mantenimiento del balance de microbios en el intestino, pero identificando los Lactobacillus dominantes ha causado mucho debate. Esto ha sido reportado que Lactobacillus fermentum (32), Lactobacillus acidophilus (33) y Lactobacillus ruminis (6) son los Lactobacillus dominante en el intestino de porcinos. Los resultados de este estudio mostro que Weisella $s p$ tuvo mayor dominancia en estómago, Pediococcus pentosaceus en intestino delgado e intestino grueso, Lactobacillus plantarum en intestino grueso y heces, mientras otras especies fueron encontradas en cada órgano.

Por lo tanto, el dominio Lactobacillus en el tracto gastrointestinal y heces del porcino fue variable en este estudio en donde la edad, dieta, tipo de crianza influye en la población microbiana (34), ya que la muestra fue aislada de un lechón destetado. Lactobacillus ruminis ha sido encontrado por ser una especie dominante en intestino humano $(35,36)$. Los resultados de este estudio indico que Lactobacillus ruminis no existe únicamente en el intestino delgado, por tanto, en el futuro, la característica de predominante de varios Lactobacillus en el tracto gastrointestinal con carácter probiótico puede ser útil en granja de porcinos.

\section{Conclusiones}

Existe una diversidad de Lactobacillus en el tracto gastrointestinal del porcino, estos microorganismos con carácter probióticos pueden ser usado como alternativa para reemplazar los antibióticos en la dieta y estimular el sistema inmunitario en los animales.

\section{Agradecimientos}

Los autores agradecen a la empresa Incabiotec y al Canon y Sobrecanon de la Universidad Nacional de Tumbes por el financiamiento del trabajo de investigación. 


\section{Referencias bibliográficas}

1. Schulze M, Nitsche-Melkus E, Hensel B, Jung M, Jakop U. Antibiotics and their alternatives in Artificial Breeding in livestock. Anim Reprod Sci. septiembre de 2020;220:106284. doi:https://doi.org/10.1016/j.anireprosci.2020.106284

2. Knecht D, Cholewińska P, Jankowska-Mąkosa A, Czyż K. Development of Swine's Digestive Tract Microbiota and Its Relation to Production Indices-A Review. Animals. 21 de marzo de 2020;10(3):527. doi:https://doi.org/10.3390/ani10030527

3. Rasschaert G, Van Elst D, Colson L, Herman L, de Carvalho Ferreira HC, Dewulf J, et al. Antibiotic residues and antibiotic-resistant bacteria in pig slurry used to fertilize agricultural fields. Antibiotics. 17 de enero de 2020;9(1):34.

doi:https://doi.org/10.3390/antibiotics9010034

4. Yang F, Hou C, Zeng X, Qiao S. The use of lactic acid bacteria as a probiotic in swine diets. Pathogens. 27 de enero de 2015;4(1):34-45.

doi:https://doi.org/10.3390/pathogens4010034

5. Kayama H, Takeda K. Manipulation of epithelial integrity and mucosal immunity by host and microbiota-derived metabolites. Eur J Immunol. 28 de julio de 2020;50(7):92131. doi:https://doi.org/10.1002/eji.201948478

6. Zheng L, Hu Y, He X, Zhao Y, Xu H. Isolation of swine-derived Lactobacillus plantarum and its synergistic antimicrobial and health-promoting properties with $\mathrm{ZnO}$ nanoparticles. J Appl Microbiol. 20 de junio de 2020;128(6):1764-75. doi:https://doi.org/10.1111/jam.14605

7. Georgieva R, Yocheva L, Tserovska L, Zhelezova G, Stefanova N, Atanasova A, et al. Antimicrobial activity and antibiotic susceptibility of Lactobacillus and Bifidobacterium spp. intended for use as starter and probiotic cultures. Biotechnol Biotechnol Equip. 2 de enero de 2015;29(1):84-91. doi:https://doi.org/10.1080/13102818.2014.987450

8. Pearlin BV, Muthuvel S, Govidasamy P, Villavan M, Alagawany M, Ragab Farag M, et al. Role of acidifiers in livestock nutrition and health: A review. J Anim Physiol Anim Nutr (Berl). 8 de marzo de 2020;104(2):558-69. doi:https://doi.org/10.1111/jpn. 13282

9. Lindsay KL, Brennan L, Kennelly MA, Maguire OC, Smith T, Curran S, et al. Impact of probiotics in women with gestational diabetes mellitus on metabolic health: a randomized controlled trial. Am J Obstet Gynecol. abril de 2015;212(4):1-11. doi:https://doi.org/10.1016/j.ajog.2015.02.008

10. Ma Z, Cheng Y, Wang S, Ge J, Shi H, Kou J. Positive effects of dietary supplementation of three probiotics on milk yield, milk composition and intestinal flora in Sannan dairy goats varied in kind of probiotics. J Anim Physiol Anim Nutr (Berl). 7 de enero de 2020;104(1):44-55. doi:https://doi.org/10.1111/jpn.13226 
11. Woodard GA, Encarnacion B, Downey JR, Peraza J, Chong K, Hernandez-Boussard T, et al. Probiotics improve outcomes after Roux-en-Y gastric bypass surgery: a prospective randomized trial. J Gastrointest Surg. 18 de julio de 2009;13(7):1198-204. doi:https://doi.org/10.1007/s11605-009-0891-X

12. Stecker RA, Moon JM, Russo TJ, Ratliff KM, Mumford PW, Jäger R, et al. Bacillus coagulans GBI-30, 6086 improves amino acid absorption from milk protein. Nutr Metab (Lond). 23 de diciembre de 2020;17(1):93. doi:https://doi.org/10.1186/s12986-02000515-2

13. Rahmdel S, Shekarforoush SS, Hosseinzadeh S, Torriani S, Gatto V. Antimicrobial spectrum activity of bacteriocinogenic Staphylococcus strains isolated from goat and sheep milk. J Dairy Sci. abril de 2019;102(4):2928-40. doi:https://doi.org/10.3168/jds.2018-15414

14. Doron S, Snydman DR. Risk and safety of probiotics. Clin Infect Dis. 15 de mayo de 2015;60:129-34. doi:https://doi.org/10.1093/cid/civ085

15. Rafter J, Bennett M, Caderni G, Clune Y, Hughes R, Karlsson PC, et al. Dietary synbiotics reduce cancer risk factors in polypectomized and colon cancer patients. Am J Clin Nutr. 1 de febrero de 2007;85(2):488-96. doi:https://doi.org/10.1093/ajcn/85.2.488

16. Bultman SJ. The microbiome and its potential as a cancer preventive intervention. Semin Oncol. febrero de 2016;43(1):97-106. doi:https://doi.org/10.1053/j.seminoncol.2015.09.001

17. Asha MZ, Khalil SFH. Efficacy and safety of probiotics, prebiotics and synbiotics in the treatment of irritable bowel syndrome: a systematic review and meta-analysis. Sultan Qaboos Univ Med J. 9 de marzo de 2020;20(1):13. doi:https://doi.org/10.18295/squmj.2020.20.01.003

18. Mohanty D, Panda S, Kumar S, Ray P. In vitro evaluation of adherence and antiinfective property of probiotic Lactobacillus plantarum DM 69 against Salmonella enterica. Microb Pathog. enero de 2019;126:212-7. doi:https://doi.org/10.1016/j.micpath.2018.11.014

19. Jeżewska-Frąckowiak J, Seroczyńska K, Banaszczyk J, Woźniak D, Żylicz-Stachula A, Skowron PM. The promises and risks of probiotic Bacillus species. Acta Biochim Pol. 6 de diciembre de 2018;65(4):509-19. doi:https://doi.org/10.18388/abp.2018_2652

20. Naghmouchi K, Belguesmia Y, Bendali F, Spano G, Seal BS, Drider D. Lactobacillus fermentum : a bacterial species with potential for food preservation and biomedical applications. Crit Rev Food Sci Nutr. 12 de noviembre de 2020;60(20):3387-99. doi:https://doi.org/10.1080/10408398.2019.1688250

21. He Y, Kim K, Kovanda L, Jinno C, Song M, Chase J, et al. Bacillus subtilis: a potential 
growth promoter in weaned pigs in comparison to carbadox. J Anim Sci. 1 de septiembre de 2020;98(9):290. doi:https://doi.org/10.1093/jas/skaa290

22. Naito S, Hayashidani H, Kaneko K, Ogawa M, Benno Y. Development of intestinal lactobacilli in normal piglets. J Appl Bacteriol. agosto de 1995;79(2):230-6. doi:https://doi.org/10.1111/j.1365-2672.1995.tb00940.x

23. Yang J, Qian K, Wang C, Wu Y. Roles of probiotic lactobacilli inclusion in helping piglets stablish healthy intestinal inter-environment for pathogen defense. Probiotics Antimicrob Proteins. 30 de junio de 2018;10(2):243-50. doi:https://doi.org/10.1007/s12602-017-9273-y

24. Mulder IE, Schmidt B, Stokes CR, Lewis M, Bailey M, Aminov RI, et al. Environmentally-acquired bacteria influence microbial diversity and natural innate immune responses at gut surfaces. BMC Biol. 2009;7(1):79. doi:https://doi.org/10.1186/1741-7007-7-79

25. Valeriano VDV, Balolong MP, Kang D-K. Probiotic roles of Lactobacillus sp. in swine: insights from gut microbiota. J Appl Microbiol. marzo de 2017;122(3):554-67. doi:https://doi.org/10.1111/jam.13364

26. Konstantinov SR, Awati AA, Williams BA, Miller BG, Jones P, Stokes CR, et al. Postnatal development of the porcine microbiota composition and activities. Environ Microbiol. julio de 2006;8(7):1191-9. doi:https://doi.org/10.1111/j.1462-

\subsubsection{X}

27. Xu C, Yan S, Guo Y, Qiao L, Ma L, Dou X, et al. Lactobacillus casei ATCC 393 alleviates Enterotoxigenic Escherichia coli K88-induced intestinal barrier dysfunction via TLRs/mast cells pathway. Life Sci. marzo de 2020;244:117281. doi:https://doi.org/10.1016/j.lfs.2020.117281

28. García V, Gambino M, Pedersen K, Haugegaard S, Olsen JE, Herrero-Fresno A. F4- and F18-positive enterotoxigenic escherichia coli isolates from diarrhea of postweaning pigs: genomic characterization. Ercolini D, editor. Appl Environ Microbiol. 10 de noviembre de 2020;86(23). doi:https://doi.org/10.1128/AEM.01913-20

29. McLoughlin S, Spillane C, Claffey N, Smith PE, O'Rourke T, Diskin MG, et al. Rumen microbiome composition is altered in sheep divergent in feed efficiency. Front Microbiol. 25 de agosto de 2020;11(1981):1-16. doi:https://doi.org/10.3389/fmicb.2020.01981

30. Lähteinen T, Lindholm A, Rinttilä T, Junnikkala S, Kant R, Pietilä TE, et al. Effect of Lactobacillus brevis ATCC 8287 as a feeding supplement on the performance and immune function of piglets. Vet Immunol Immunopathol. marzo de 2014;158(1-2):1425. doi:https://doi.org/10.1016/j.vetimm.2013.09.002 
31. Zhang L, Liu S, Li M, Piao X. Effects of maternal 25-hydroxycholecalciferol during the last week of gestation and lactation on serum parameters, intestinal morphology and microbiota in suckling piglets. Arch Anim Nutr. 1 de noviembre de 2020;74(6):445-61. doi:https://doi.org/10.1080/1745039X.2020.1822710

32. Alqazlan N, Astill J, Taha-Abdelaziz K, Nagy É, Bridle B, Sharif S. Probiotic lactobacilli enhance immunogenicity of an inactivated H9N2 influenza virus vaccine in chickens. Viral Immunol. 1 de marzo de 2021;34(2):86-95. doi:https://doi.org/10.1089/vim.2020.0209

33. Yan F, Polk DB. Probiotics and immune health. Curr Opin Gastroenterol. noviembre de 2011;27(6):496-501. doi:https://doi.org/10.1097/MOG.0b013e32834baa4d

34. Alasmary F, Snelling A, Zain M, Alafeefy A, Awaad A, Karodia N. Synthesis and evaluation of selected benzimidazole derivatives as potential antimicrobial agents. Molecules. 20 de agosto de 2015;20(8):15206-23. doi:https://doi.org/10.3390/molecules200815206

35. Sugiharto S, Ranjitkar S. Recent advances in fermented feeds towards improved broiler chicken performance, gastrointestinal tract microecology and immune responses: A review. Anim Nutr. marzo de 2019;5(1):1-10. doi:https://doi.org/10.1016/j.aninu.2018.11.001

36. Wilkins T, Sequoia J. Probiotics for gastrointestinal conditions: a summary of the evidence. Am Fam Physician [Internet]. 2017;96(3):170-9. Disponible en: https://www.aafp.org/afp/2017/0801/p170.html

\section{Conflicto de intereses}

Los autores declaramos que no existen conflictos de interés.

\section{Contribuciones de los autores}

Fabian-Dominguez, Fredy; Vásquez-Rojas, Lourdes; Baylon-Cuba Miluska y López-Flores, Alicia: desarrollaron la parte experimental con animales, procesamiento de datos y redacción del manuscrito.

Mialhe Eric: diseño del estudio de investigación. 